\title{
The Minimum Size of Digraphs Satisfying Directed Cut Conditions
}

\author{
Huawen Ma \\ College of Mathematics and Computer Science, Yan'an University, Yan'an 716000, Shaanxi, China \\ Correspondence should be addressed to Huawen Ma; mhwaue@sina.com
}

Received 3 December 2021; Revised 15 January 2022; Accepted 19 January 2022; Published 14 February 2022

Academic Editor: Guozeng Cui

Copyright $(0) 2022$ Huawen Ma. This is an open access article distributed under the Creative Commons Attribution License, which permits unrestricted use, distribution, and reproduction in any medium, provided the original work is properly cited.

A directed graph (digraph) $D$ of order $n$ satisfies directed cut condition $(D C C)$ if there are at least $|S|$ arcs from any set $S \subseteq V(D)$, $|S| \leq(n / 2)$ to its complement $\bar{S}=V(D) / S$. We show that a digraph $D$ of even order $n$ has at least $2 n-3$ arcs if $D$ satisfies $D C C$.

\section{Introduction}

A network is made up of nodes and links between nodes. Suppose that a cluster of $k$ hosts in one part of the network wishes to communicate with $k$ hosts at another part of the network. Assume that any two communication paths can use common intermediate nodes, but no two communication paths between distinct pairs can share common links. Then, there must exist at least $k$ links leaving the cluster. A simple graph can quite naturally represent this communication network. Path-pairability is a notion that emerged from this practical networking problem introduced by Csaba et al. [1] and further studied by Faudree et al. [2-4]. A graph $G$ of even order $n$ is path-pairable, if for any set of disjoint pairs $\left\{s_{i}, t_{i}\right\}, \quad 1 \leq i \leq(n / 2), \quad G$ has pairwise edge disjoint $\left(s_{i}, t_{i}\right)$-paths. A series of problems on path-pairable graphs have been proposed, such as seeking diameter, maximum order, and maximum degree. In [4], Faudree et al. considered the problem of investigating the minimum size of path-pairable graph $G$ of order $n$. It is trivial that $|E(G)| \geq n-1$ as $K_{1, n-1}$ is path-pairable. They showed that $|E(G)| \geq(3 n / 2)-\log n-O(1)$ for path-pairable graph $G$ with $\Delta(G)<n-1$.

A graph on $n$ vertices satisfies cut condition (CC), if for any $S \subseteq V(G),|S| \leq(n / 2)$, there are at least $|S|$ edges between $S$ and $\bar{S}=V(G) / S$. Obviously, a path-pairable graph satisfies $C C$, but the inverse is not. Jobson et al. [5] proved that a graph $G$ of even order $n$ with $\Delta(G)<n-1$, if $G$ satisfies $C C$, then $|E(G)| \geq(3 n / 2-3)$, and this bound is tight. A graph of even order $n$ satisfies even cut condition (ECC), if for any $S \subseteq V(G),|S|=(n / 2)$, there are at least $(n / 2)$ edges between $S$ and $\bar{S}$. Jobson et al. [5] showed that if $G$ is a graph of order $n \equiv 0(\bmod 4)$ with $\Delta(G)<n-1$ and satisfies ECC, then $|E(G)| \geq(5 n / 4-2)$.

On account of the results of Jobson et al., we consider the minimum size of the digraphs satisfying directed cut condition in this paper. Let $D$ be a digraph with vertex set $V(D)$ and arc set $E(D)$. For any two disjoint sets $A, B \subseteq V(D)$, let $e(A, B)$ denote the number of arcs from $A$ to $B$ in $D$. We say a digraph $D$ on $n$ vertices satisfies directed cut condition $(D C C)$, if $e(S, \bar{S}) \geq|S|$ for any $S \subseteq V(D)$ with $|S| \leq(n / 2)$.

Let $D_{1}$ be a digraph with $n$ vertices and $V\left(D_{1}\right)=\{u\} \cup S$, where $S$ is an independent set with $n-1$ vertices in $D_{1}$. In $D_{1}$, $u$ directs to each vertex of $S$ and each vertex in $S$ directs to $u$. Let $D_{2}$ be a digraph with $n$ (even) vertices and $V\left(D_{2}\right)=$ $\{u\} \cup\{v\} \cup S \cup T$, where $S$ and $T$ are two independent sets with $(n / 2)-1$ vertices in $D_{2}$. In $D_{2}, u$ directs to each vertex of $S$ and each vertex in $S$ directs to $v ; v$ directs to each vertex of $T$ and each vertex in $T$ directs to $u ; u$ directs to $v$ and $v$ directs to $u$. It is easy to verify that $D_{1}$ and $D_{2}$ with size $2 n-2$ arcs satisfy $D C C$, which implies that the minimum size of digraphs satisfying $D C C$ is at most $2 n-2$, whether the maximum degree is $2 n-2$ or not. The following theorem gives that each digraph with $n$ (even) vertices satisfying DCC has at least $2 n-3$ arcs.

Theorem 1.1. Let $D$ be a digraph of even order $n$. If $D$ satisfies DCC, then $D$ has at least $2 n-3$ arcs. 
Remark 1.2. We do not know whether there is a digraph which has $2 n-3$ arcs and satisfy DCC. But by the digraphs $D_{1}$ and $D_{2}$, we know $2 n-3$ or $2 n-2$ is the tight value.

Corresponding to the ECC of undirected graphs, we say a digraph $D$ of even order $n$ satisfies evendirected cut condition (EDCC), if $e(S, \bar{S}) \geq(n / 2)$ for any $S \subseteq V(D)$ with $|S|=(n / 2)$.

Theorem 1.3. Let $D$ be a digraph of even order $n$. If $D$ satisfies EDCC, then $D$ has at least $2 n-\sqrt{n}$ arcs.

We say a digraph $D$ satisfies weakly even directed cut condition (WEDCC), if $\max \{e(S, \bar{S}), e(\bar{S}, S)\} \geq(n / 2)$ for any $S \subseteq V(D)$ with $|S|=(n / 2)$. Let $D_{3}$ be an orientation of $K_{1, n-1}$ such that the apex directs to other vertices. Clearly, $D_{3}$ has $n-1$ arcs and satisfies WEDCC. Hence, we limit the maximum degree of digraphs. Using the probabilistic method, we give the following result.

Theorem 1.4. Let $D$ be a digraph of even order $n$. For any $0<\epsilon \leq 1$, if $\Delta(D) \leq \epsilon^{2}(n / 128)$ and $D$ satisfies WEDCC, then $D$ has at least $(2 n-2 \sqrt{n} / 1+\epsilon)$ arcs.

The rest of this paper is organized as follows. In the next section, we state some notations and lemmas used. In Section 3, we give a proof of Theorem 1.1. In Section 4, we prove Theorems 1.3 and 1.4. The final section contains some concluding remarks.

\section{Notations and Lemmas}

We consider digraphs without loops and parallel arcs, but the reverse parallel arcs are allowed. We first introduce some notations and definitions. Let $D$ be a digraph with vertex set $V(D)$ and arc set $E(D)$, and let $e(D)=|E(D)|$. Given $x, y \in V(D)$, we write $x y$ for the arc directed from $x$ to $y$. We call vertex $y$ as an outneighbour of $x$ and $x$ as an inneighbour of $y$. For any vertex $v \in V(D)$, let $N_{D}^{+}(v)$ be the outneighbours set of $v$ in $D$, and let $d_{D}^{+}(v)=\left|N_{D}^{+}(v)\right|$ be the outdegree of $v$. Similarly, we write $N_{D}^{-}(v)$ as the in-neighbours set of $v$ in $D$ and $d_{D}^{-}(v)=$ $\left|N_{D}^{-}(v)\right|$ as the indegree of $v$. Let $N_{D}(v)=N_{D}^{+}(v) \cup N_{D}^{-}(v)$ be the set of neighbours of $v$ and $d_{D}(v)=d_{D}^{+}(v)+d_{D}^{-}(v)$ be degree of $v$. Let $\Delta^{+}(D), \Delta^{-}(D), \Delta(D)$, and $\delta(D)$ be the maximum outdegree, maximumindegree, maximum degree, and minimum degree of $D$, respectively. When understood, the subscript may be dropped. For any digraph $D$, we can associate a simple graph $G$ on the same vertex set by replacing each arc by an edge and deleting parallel edges if there are. Such a simple graph $G$ is called underlying graph of $D$. We also call as digraph $D k$-connected if its underlying graph $G$ is $k$-connected. For a digraph $D$, we call $\left(V_{1}, V_{2}\right)$ a partition of $D$ if $V_{1} \cup V_{2}=V(D)$ and $V_{1} \cap V_{2}=\varnothing$. Let $e\left(V_{1}, V_{2}\right)$ denote the number of arcs from $V_{1}$ to $V_{2}$ and $e\left(V_{i}\right)$ be the number of arcs such that two ends lie in $V_{i}, i=1,2$. Let $D\left[V_{i}\right]$ denote the induced subdigraph of $V_{i}$.

Now, we state several theorems which will be used in the following proofs. The first one is a well-known result on partitioning due to Lóvasz [6]: if $G$ is a 2-connected graph of order $a_{1}+a_{2}$, where $a_{1}, a_{2}$ are positive integers, then $G$ has a partition $V(G)=A_{1} \cup A_{2}$ such that $A_{i}$ induces a connected subgraph of order $a_{i}, i=1,2$. The following result introduced in [7] is stronger than Lóvasz's, which plays a key role in our proofs.

Lemma 2.1 (see [7]). If $G$ is a 2-connected graph of order $n$, then $V(G)$ has a labeling $v_{1}, v_{2}, \ldots, v_{n}$ such that for every $1<i<n, v_{i}$ has a neighbour in both $\left\{v_{1}, \ldots, v_{i-1}\right\}$ and $\left\{v_{i+1}, \ldots, v_{n}\right\}$.

A bisection of a graph $G$ is a partition of $V(G)=V_{1} \cup V_{2}$ such that $\left\|V_{1}|-| V_{2}\right\| \leq 1$. We use $G^{c}$ to denote the complement of a graph $G$. The next lemma on minimum bisection due to Fan et al. [8] has strong correlation with our problems.

Lemma 2.2 (see [8]). Let $M$ be a maximum matching in $G^{c}$ of a graph $G$ which has $n$ vertices and $m$ edges. Then, $G$ admits a bisection $V_{1}, V_{2}$ such that $\left(V_{1}, V_{2}\right) \leq(m+\lfloor n / 2\rfloor-|M|) / 2$.

We introduce the well-known Azuma-Hoeffding inequality $[9,10]$ and use the version given in the book (see Corollary 2.27) of Janson et al. [11].

Lemma 2.3 (see [11]). Let $Z_{1}, Z_{2}, \ldots, Z_{n}$ be independent random variables taking values in $\{1,2, \ldots, k\}$, let $Z:=\left(Z_{1}, Z_{2}, \ldots, Z_{n}\right)$, and let $f:\{1,2, \ldots, k\} \longrightarrow N$ such that $\left|f(Y)-f\left(Y^{\prime}\right)\right| \leq c_{i}$ for any $Y, Y^{\prime} \in\{1,2, \ldots, k\}^{n}$ which differ only in the ith coordinate. Then, for any $z>0$,

$$
\begin{aligned}
& \operatorname{Pr}(f(Z) \geq E(f(Z))+z) \leq \exp \left(\frac{-z^{2}}{2 \sum_{i=1}^{n} c_{i}^{2}}\right), \\
& \operatorname{Pr}(f(Z) \leq E(f(Z))-z) \leq \exp \left(\frac{-z^{2}}{2 \sum_{i=1}^{n} c_{i}^{2}}\right) .
\end{aligned}
$$

\section{Proof of Theorem 1.1}

Proof. Let $D$ be a digraph of $n$ (even) vertices satisfying $D C C$. Suppose on the contrary that $e(D)<2 n-3$. We may suppose that $D$ is not 2-connected. For otherwise, by Lóvasz's theorem, $D$ has a bisection $V_{1}, V_{2}$ such that $D\left[V_{1}\right]$ and $D\left[V_{2}\right]$ induce two connected subdigraphs, respectively. By DCC, $e\left(V_{1}, V_{2}\right) \geq(n / 2)$ and $e\left(V_{2}, V_{1}\right) \geq(n / 2)$. So,

$$
\begin{aligned}
e(D) & \geq e\left(V_{1}\right)+e\left(V_{2}\right)+e\left(V_{1}, V_{2}\right)+e\left(V_{2}, V_{1}\right) \\
& \geq 2 \times\left(\frac{n}{2}-1\right)+2 \times \frac{n}{2} \geq 2 n-2,
\end{aligned}
$$

a contradiction.

Since $D$ is not 2-connected, $D$ has cut vertices. Let $u$ be a cut vertex of $D$ and $C_{1}, C_{2}, \ldots, C_{k}(k \geq 2)$ be connected components of $D-u$. Then, by DCC, we have a simple fact: for any $C_{i}$ with $\left|C_{i}\right| \leq(n / 2)$, and for any $v \in V\left(C_{i}\right), v u \in E(D)$.

We first claim that $d^{-}(u) \neq n-1$. Suppose for the contradiction that $d^{-}(u)=n-1$, if there exists a component, say $C_{1}$, with order larger than $n / 2$, then $e\left(V(D) \backslash V\left(C_{1}\right), V\left(C_{1}\right)\right)=e\left(u, V\left(C_{1}\right)\right) \geq n-\left|C_{1}\right|$ by DCC. Thus, 


$$
\begin{aligned}
e(D) & \geq e\left(C_{1}\right)+\sum_{i=2}^{k} e\left(C_{i}\right)+e\left(V(D), V\left(C_{1}\right), V\left(C_{1}\right)\right)+d^{-}(u) \\
& \geq\left(\left|C_{1}\right|-1\right)+\left(n-\left|C_{1}\right|\right)+n-1=2 n-2
\end{aligned}
$$

a contradiction. So, we suppose that $\left|C_{i}\right| \leq n / 2(1 \leq i \leq k)$. Without loss of generality, let $\left|C_{k}\right|=\max \left\{\left|C_{1}\right|,\left|C_{2}\right|\right.$, $\left.\ldots,\left|C_{k}\right|\right\}$. We split $\left\{C_{1}, C_{2}, \ldots, C_{k-1}\right\}=A_{1} \cup A_{2}$ such that $\left|A_{1}\right|$ and $\left|A_{2}\right|$ are as close as possible. Then, we partition $V\left(C_{k}\right)-v$ into two sets $B_{1}, B_{2}$ such that $\left|A_{1}\right|+\left|B_{1}\right|=$ $(n / 2)-1$, where $v$ is an arbitrary vertex of $C_{k}$. Let $E_{1}=A_{1}$ $\cup B_{1}, E_{2}=A_{2} \cup B_{2}$. Let $a_{1}=e\left(u, E_{1}\right), a_{2}=e\left(u, E_{2}\right), b_{1}=e$ $\left(B_{1}, B_{2}\right), \quad b_{2}=e\left(B_{2}, B_{1}\right), c_{1}=e\left(B_{1}, v\right)$, and $c_{2}=e\left(B_{2}, v\right)$. Define

$$
d= \begin{cases}0, & u v \in E(D) \\ 1, & \text { otherwise. }\end{cases}
$$

By DCC, for bisections $V(D)=\left(E_{1} \cup u, E_{2} \cup v\right)$ and $V(D)=\left(E_{2} \cup u, E_{1} \cup v\right)$, we have

$$
\begin{aligned}
e( & \left.E_{1} \cup u, E_{2} \cup v\right) \\
& =e\left(u, E_{2}\right)+e\left(B_{1}, B_{2}\right)+e\left(B_{1}, v\right)+d \\
& =a_{2}+b_{1}+c_{1}+d \geq \frac{n}{2} .
\end{aligned}
$$

Similarly,

$$
\begin{aligned}
e( & \left.E_{2} \cup u, E_{1} \cup v\right) \\
& =e\left(u, E_{1}\right)+e\left(B_{2}, B_{1}\right)+e\left(B_{2}, v\right)+d \\
& =a_{1}+b_{2}+c_{2}+d \geq \frac{n}{2} .
\end{aligned}
$$

Adding up (5) and (6), we obtain that

$$
a_{1}+a_{2}+b_{1}+b_{2}+c_{1}+c_{2} \geq n-2 \text {. }
$$

Thus, $e(D) \geq d^{-}(u)+a_{1}+a_{2}+b_{1}+b_{2}+c_{1}+c_{2} \geq 2 n-3$, a contradiction.

Now, we suppose that $d^{-}(u)<n-1$. Then, there is exact one component of $D-u$ which has order larger than $(n / 2)$. We call such a subdigraph the large component at $u$ and denote it by $L(u)$. We claim that all cut vertices of $D$ are contained in one block; we call it central block of $D$. Assume it is not true. Then, there must exist cut vertices $x_{1}, x_{2}$, and $x_{3}$ and blocks $B_{1}, B_{2}, B_{3}$, and $B_{4}$ such that $x_{i} \in B_{i} \cap B_{i+1}$ for $i=1,2,3 . B_{1}$ and $B_{4}$ belong to different components of $D-x_{2}$, and then there must exist a block which does not belong to the large component at $x_{2}$, say $B_{1}$. That is to say, each vertex of the component containing $B_{1}$ directs to $x_{2}$ by the fact we mentioned above. Then, removing the cut vertex $x_{1}$ cannot separate $B_{1}$ and $B_{2}$, a contradiction.

Let $B$ be the central block of $D$ (if $D$ has only one cut vertex $u$, then let $B:=L(u)+u$.). We define a weight for each vertex $x$ of $B$ as follows:

$$
\omega(x)= \begin{cases}|V(D)-V(L(x))|, & \text { if } x \text { is a cut vertex } \\ 1, & \text { otherwise }\end{cases}
$$

By the definition of $\omega(x)$, it is easy to see $\omega(x)<(n / 2)$ for each $x \in B$.

Since $B$ is a block of $D$, by Lemma $2.1, B$ has a labeling $v_{1}, v_{2}, \ldots, v_{|B|}$ such that for each $1<i<|B|, v_{i}$ has a neighbour in both sets $\left\{v_{1}, \ldots, v_{i-1}\right\}$ and $\left\{v_{i+1}, \ldots, v_{|B|}\right\}$. Choose the smallest number $k$ such that $w_{1}=\sum_{i=1}^{k-1} \omega\left(v_{i}\right) \leq(n / 2)$ and $\sum_{i=1}^{k} \omega\left(v_{i}\right)=w_{1}+\omega\left(v_{k}\right)>(n / 2)$. Let

$W_{1}=\cup\left\{V\left(B^{\prime}\right) \mid B^{\prime} \neq B\right.$ is a block and $\left.B^{\prime} \cap\left\{v_{1}, \ldots, v_{k-1}\right\} \neq \varnothing\right\}$.

According to the definition of weight value of vertices of $B$, we can easily find that $\left|W_{1}\right|=w_{1}$.

We claim that $w_{1}=\left|W_{1}\right|<(n / 2)$. For otherwise, partition $V(D)$ into two sets, $W_{1}$ and $V(D) \backslash W_{1}$, which induce two connected subdigraphs by Lemma 2.1. Thus, by DCC, we have

$$
\begin{aligned}
e(D) & =e\left(W_{1}\right)+e\left(V(D) \backslash W_{1}\right)+e\left(W_{1}, V(D), W_{1}\right)+e\left(V(D), W_{1}, W_{1}\right), \\
& \geq 2 \times\left(\frac{n}{2}-1\right)+2 \times \frac{n}{2} \geq 2 n-2,
\end{aligned}
$$

a contradiction.

We have $\omega\left(v_{k}\right) \geq 2$ as $w_{1}<(n / 2)$ and $w_{1}+\omega\left(v_{k}\right)>n / 2$. That is to say, $v_{k}$ is a cut vertex of $D$. Let $W_{2}=\cup\left\{V\left(B^{\prime}\right) \mid B^{\prime} \neq B\right.$ is a block and $\left.B^{\prime} \cap\left\{v_{k+1}, \ldots, v_{|B|}\right\}\right\} \neq \varnothing$.

Then, $W_{2} \neq \varnothing$ as $w_{1}+\omega\left(v_{k}\right)<n$. Let $W_{0}=V(D) /$ $\left(W_{1} \cup W_{2}\right)$, then blocks containing $v_{k}$ are contained in $W_{0}$. Let $v_{k}=u$ and $v$ be an arbitrary vertex of $W_{0} \backslash\{u\}$. Partition
$W_{0} /\{u, v\}$ into two sets $W_{0}^{1}, W_{0}^{2}$ such that $\left|W_{0}^{1}\right|+\left|W_{1}\right|=n / 2-1$, then $\left|W_{0}^{2}\right|+\left|W_{2}\right|=n / 2-1$.

Let $\quad e\left(u, W_{1} \cup W_{0}^{1}\right)=\alpha_{1}, \quad e\left(u, W_{2} \cup W_{0}^{2}\right)=\alpha_{2}$; let $e\left(W_{1} \cup W_{0}^{1}, u\right)=\beta_{1}, \quad e\left(W_{2} \cup W_{0}^{2}, u\right)=\beta_{2} ; \quad$ let $\quad e\left(W_{1} \cup\right.$ $\left.W_{0}^{1}, W_{2} \cup W_{0}^{2}\right)=e\left(W_{0}^{1}, W_{0}^{2}\right)=\gamma_{1}, \quad e\left(W_{2} \cup W_{0}^{2}, W_{1} \cup W_{0}^{1}\right)$ $=e\left(W_{0}^{2}, W_{0}^{1}\right)=\gamma_{2}$; let $e\left(v, W_{0}^{1}\right)=\delta_{1}, e\left(v, W_{0}^{2}\right)=\delta_{2}$; and let $e\left(W_{0}^{1}, v\right)=\theta_{1}, e\left(W_{0}^{2}, v\right)=\theta_{2}$.

For bisections $V(D)=\left(W_{1} \cup W_{0}^{1} \cup u, W_{2} \cup W_{0}^{2} \cup v\right)$ and $V(D)=\left(W_{1} \cup W_{0}^{1} \cup v, W_{2} \cup W_{0}^{2} \cup u\right)$, by $D C C$, we have 


$$
\begin{aligned}
& e\left(W_{1} \cup W_{0}^{1} \cup u, W_{2} \cup W_{0}^{2} \cup v\right) \\
& =e\left(W_{1} \cup W_{0}^{1}, W_{2} \cup W_{0}^{2}\right)+e\left(W_{1} \cup W_{0}^{1}, v\right)+e\left(u, W_{2} \cup W_{0}^{2}\right)+d \\
& \quad=\gamma_{1}+\theta_{1}+\alpha_{2}+d \geq \frac{n}{2}, \\
& e\left(W_{2} \cup W_{0}^{2} \cup v, W_{1} \cup W_{0}^{1} \cup u\right) \\
& \quad=e\left(W_{2} \cup W_{0}^{2}, W_{1} \cup W_{0}^{1}\right)+e\left(W_{2} \cup W_{0}^{2}, u\right)+e\left(v, W_{1} \cup W_{0}^{1}\right)+1 \\
& \quad=\gamma_{2}+\beta_{2}+\delta_{1}+1 \geq \frac{n}{2}, \\
& e\left(W_{1} \cup W_{0}^{1} \cup v, W_{2} \cup W_{0}^{2} \cup u\right) \\
& \quad=e\left(W_{1} \cup W_{0}^{1}, W_{2} \cup W_{0}^{2}\right)+e\left(W_{1} \cup W_{0}^{1}, u\right)+e\left(v, W_{2} \cup W_{0}^{2}\right)+1 \\
& \quad=\gamma_{1}+\beta_{1}+\delta_{2}+1 \geq \frac{n}{2},
\end{aligned}
$$

and

$$
\begin{aligned}
& e\left(W_{2} \cup W_{0}^{2} \cup u, W_{1} \cup W_{0}^{1} \cup v\right) \\
& \quad=e\left(W_{2} \cup W_{0}^{2}, W_{1} \cup W_{0}^{1}\right)+e\left(W_{2} \cup W_{0}^{2}, v\right)+e\left(u, W_{1} \cup W_{0}^{1}\right)+d \\
& \quad=\gamma_{2}+\theta_{2}+\alpha_{1}+d \geq \frac{n}{2} \\
& \quad e\left(W_{0}, V(D), W_{0}\right) \geq\left|W_{0}\right|=\left|W_{0}^{1}\right|+\left|W_{0}^{2}\right|+2 .
\end{aligned}
$$

Adding up (12) and (13), we obtain that

$$
\begin{aligned}
& \alpha_{1}+\alpha_{2}+\beta_{1}+\beta_{2}+\gamma_{1}+\gamma_{2}+\delta_{1}+\delta_{2}+\theta_{1}+\theta_{2} \\
\geq & 2 n-2-2 d-\left(\gamma_{1}+\gamma_{2}\right) .
\end{aligned}
$$

Recall that $\omega\left(v_{k}\right)<(n / 2)$, then $\left|W_{0}\right| \leq(n / 2)$. Considering the partition $V(D)=\left(W_{0}, V(D) \backslash W_{0}\right)$, by $D C C$, we have
Note that the subdigraphs induced by $W_{i}$ for $i=1,2$ are connected and each vertex of $W_{0} \backslash\{u\}$ directs to $u$. Thus, by (14), we have

$$
\begin{aligned}
e(D) & \geq\left(\left|W_{1}\right|-1\right)+\left(\left|W_{2}\right|-1\right)+\left(\left|W_{0}\right|-1\right)+\sum_{i=1}^{2}\left(\alpha_{i}+\left(\beta_{i}-\left|W_{0}^{i}\right|\right)+\gamma_{i}+\theta_{i}+\delta_{i}\right)+d \\
& \geq(n-3)+2 n-2-2 d-\left(\gamma_{1}+\gamma_{2}\right)-\left(\left|W_{0}\right|-2\right)+d \\
& \geq 2 n-3+\left(n-d-\left|W_{0}\right|-\gamma_{1}-\gamma_{2}\right) .
\end{aligned}
$$

If $\left|W_{0}\right|+\gamma_{1}+\gamma_{2} \leq n-1$, then $e(D) \geq 2 n-3$, we are done. So, we suppose that $\left|W_{0}\right|+\gamma_{1}+\gamma_{2} \geq n$. Thus, 


$$
\begin{aligned}
e(D) & \geq\left(\left|W_{1}\right|-1\right)+\left(\left|W_{2}\right|-1\right)+e\left(W_{0},\{u\}, u\right)+e\left(u, W_{1} \cup W_{2}\right)+\gamma_{1}+\gamma_{2} \\
& \geq\left(\left|W_{1}\right|-1\right)+\left(\left|W_{2}\right|-1\right)+\left(\left|W_{0}\right|-1\right)+\left|W_{0}\right|+\gamma_{1}+\gamma_{2} \\
& \geq n-3+n=2 n-3,
\end{aligned}
$$

a contradiction too.

\section{Proofs of Theorems 1.3 and 1.4}

Proof of Theorem 1.3. Let $D$ be a digraph with $n$ vertices which satisfies EDCC, and let $G$ be the underlying graph of $D$ and $M$ be the maximum matching of $G^{c}$. We need to show that $e(D) \geq 2 n-\sqrt{n}$. We may suppose that $|M| \geq(n-$ $2 \sqrt{n}+1) / 2$. For otherwise, we have $\omega(G)=n-2|M| \geq 2 \sqrt{n}$. Hence, $e(D) \geq e(G) \geq(2 \sqrt{n})(2 \sqrt{n}-1) / 2=2 n-\sqrt{n}$, we are done.

Suppose on the contrary that $e(D)<2 n-\sqrt{n}$. Then, by Lemma 2.2,D admits a bisection $V(D)=V_{1} \cup V_{2}$ such that

$$
\begin{aligned}
e\left(V_{1}, V_{2}\right) & \leq \frac{(e(D)+(n / 2)-|M|)}{4} \\
& <\frac{(2 n-\sqrt{n}+(n / 2)-(n-2 \sqrt{n}+1) / 2)}{4} \\
& <\left(\frac{n}{2}\right),
\end{aligned}
$$

a contradiction with EDCC.

Now, we prove Theorem 1.4 using the probabilistic method.

Lemma 4.1. Let $D$ be a digraph with $n$ (even) vertices and $m$ arcs, $G$ be the underlying graph of $D$, and $M$ be a maximum matching of $G^{c}$, then $D$ admits a bisection $V(D)=V_{1} \cup V_{2}$ such that

$$
\max \left\{e\left(V_{1}, V_{2}\right), e\left(V_{2}, V_{1}\right)\right\} \leq \frac{(m / 4+n / 4)}{(\dashv M \mid / 2)}+R
$$

where $R=2 \sqrt{\sum_{v \in V(D)} d^{2}(v)}$.

Proof. Let $M=\left\{u_{1} v_{1}, u_{2} v_{2}, \ldots, u_{(|M| / 2)} v_{(|M| / 2)}\right\}$. We know $G-V(M)$ is a clique, as $M$ is a maximum matching of $G^{c}$. Let $u_{|(M / 2+1)|} v_{|(M / 2+1)|}, \ldots, u_{(n / 2)} v_{(n / 2)}$ be a perfect matching of $G-V(M)$. Partition $V(D)$ into $(n / 2)$ disjoint pairs $\left\{u_{1}, v_{1}\right\},\left\{u_{2}, v_{2}\right\}, \ldots,\left\{u_{(n / 2)}, v_{(n / 2)}\right\}$. Let $Z=\left(Z_{1}, Z_{2}, \ldots\right.$, $\left.Z_{(n / 2)}\right)$ be a random, independent, and uniform 2-coloring (with color 1 and 2) of $\left\{u_{1}, u_{2}, \ldots, u_{(n / 2)}\right\}$, and color each $v_{i}$ with $3-Z_{i}$ for $1 \leq i \leq(n / 2)$. For $i=1,2$, let

$$
V_{i}=\{v \in V(D) \mid v \text { was colored } i\} .
$$

Thus, $V(D)=V_{1} \cup V_{2}$ is a bisection of $D$.

Now, we bound $E\left(e\left(V_{1}, V_{2}\right)\right)$. For each arc $e=u v \in E(D)$, let $I_{e}$ be the indicator random variable of the event $e \in E\left(V_{1}, V_{2}\right)$. If the endpoints $u, v$ of $e$ were paired in the beginning, i.e., $\{u, v\}=\left\{u_{i}, v_{i}\right\}$ for some $(|M| / 2)$ $<i \leq(n / 2)$, then $P_{r}\left(e \in E\left(V_{1}, V_{2}\right)\right)=(1 / 2)$. Otherwise,

$$
\begin{aligned}
P_{r}\left(e \in E\left(V_{1}, V_{2}\right)\right) & =P_{r}\left(u \in V_{1}, v \in V_{2}\right) \\
& =P_{r}\left(u \in V_{1}\right) P_{r}\left(v \in V_{2}\right)=\left(\frac{1}{4}\right) .
\end{aligned}
$$

Obviously, there are at most $2 \times((n / 2)-|M|)$ arcs which were paired in the beginning. By linearity of expectation, we have

$$
\begin{aligned}
E\left(e\left(V_{1}, V_{2}\right)\right) & =\sum_{e \in E(D)} E\left(I_{e}\right) \leq \frac{(n-2|M|)}{2}+\frac{(m-n+2|M|)}{4} \\
& =\frac{m}{4}+\frac{n}{4}-\frac{|M|}{2} .
\end{aligned}
$$

By symmetry, we also have $E\left(e\left(V_{2}, V_{1}\right)\right) \leq(m / 4)$ $+(n / 4)-(|M| / 2)$. Note that changing the color of any vertex $u_{i}$ for $1 \leq i \leq(n / 2)$ cannot affect $\left\{e\left(V_{1}, V_{2}\right), e\left(V_{2}, V_{1}\right)\right\}$ by more than $d\left(u_{i}\right)+d\left(v_{i}\right)$. Define

$$
L=\sum_{i=1}^{(n / 2)}\left(d\left(u_{i}\right)+d\left(v_{i}\right)\right)^{2}
$$

Then, we have

$$
L \leq 2 \sum_{i=1}^{(n / 2)}\left(d^{2}\left(u_{i}\right)+d^{2}\left(v_{i}\right)\right)=2 \sum_{v \in V(D)} d^{2}(v) .
$$

By Lemma 2.3, we obtain

$P_{r}\left(e\left(V_{1}, V_{2}\right) \geq E\left(e\left(V_{1}, V_{2}\right)\right)+R\right) \leq \exp \left(-\frac{R^{2}}{2 L}\right) \leq e^{-1}$,

and

$P_{r}\left(e\left(V_{2}, V_{1}\right) \geq E\left(e\left(V_{2}, V_{1}\right)\right)+R\right) \leq \exp \left(-\frac{R^{2}}{2 L}\right) \leq e^{-1}$.

Hence, there is a bisection $V(D)=V_{1} \cup V_{2}$ of $D$ such that

$$
\max \left\{e\left(V_{1}, V_{2}\right), e\left(V_{2}, V_{1}\right)\right\} \leq \frac{m}{4}+\frac{n}{4}-\frac{|M|}{2}+R .
$$

According to Lemma 4.1, we can easily obtain a fact: if $m<n+2|M|-4 R$, then $D$ admits a bisection $V(D)=$ $V_{1} \cup V_{2}$ such that $\max \left\{e\left(V_{1}, V_{2}\right), e\left(V_{2}, V_{1}\right)\right\}<(n / 2)$.

Lemma 4.2. Let $D$ be a digraph with $n$ (even) vertices and $m \geq n$ arcs. Let $G$ be the underlying graph of $D$ and $M$ be a maximum matching of $G^{c}$. For $0<\epsilon \leq 1$, if $\Delta \leq \epsilon^{2}(n / 128)$ and $m<(n+2|M| / 1+\epsilon), \quad$ then $\max \left\{e\left(V_{1}, V_{2}\right), e\left(V_{2}, V_{1}\right)\right\}<$ $(n / 2)$. 
Proof. Note that $R=2 \sqrt{\sum_{v \in V(D)} d^{2}(v)} \leq 2 \sqrt{\Delta \sum_{v \in V(D)} d(v)}$ $=(2 \sqrt{2 \Delta m} \leq \epsilon m / 4)$, since $m<n+2|M| / 1+\epsilon$, which implies $m<n+2|M|-\epsilon m<n+2|M|-4 R$. By the fact mentioned above, we have $\max \left\{e\left(V_{1}, V_{2}\right), e\left(V_{2}, V_{1}\right)\right\}<(n / 2)$.

Proof of Theorem 1.4. We may suppose that $|M|>(n / 2)-\sqrt{n}$. For otherwise, $|V(G)-V(M)| \geq 2 \sqrt{n}$ and $V(G)-V(M)$ is a clique. Hence,

$$
e(D) \geq e(G) \geq e(G-V(M))=\frac{2 \sqrt{n}(2 \sqrt{n}-1)}{2}=2 n-\sqrt{n} .
$$

We are done. Since $|M|>(n / 2)-\sqrt{n}$, by Lemma 4.2 and $W E D C C$, we have $e(D) \geq(n+2|M| / 1+\epsilon) \geq(2 n-2 \sqrt{n} /$ $1+\epsilon)$.

\section{Concluding Remarks and Open Problems}

We in this paper consider extremum problems in directed graphs and give some lower bounds of arcs when a digraph satisfies DCC, EDCC, and WEDCC, respectively. Below, we give some comments on these results and propose some open problems.

For Theorem $1.1,2 n-3$ or $2 n-2$ could be the exact value although we do not know which one to be. Considering the symmetry of this condition, we prefer to believe that it is an even number. Hence, we propose the following conjecture.

Conjecture 5.1. Let $D$ be a digraph of order $n$. If $D$ satisfies $D C C$, then $e(D) \geq 2 n-2$.

For Theorem 1.3, it is a corollary of Lemma 2.2. In fact, $E D C C$ is closely related to the minimum cut. Obviously, if a digraph with maximum indegree or outdegree $n-1$, then it must satisfy $W E D C C$. It is not clear to us what the exact value is when the maximum indegree and outdegree are smaller than $n-1$.

Problem 5.2. What is the minimum size of a digraph which satisfies EDCC?

Problem 5.3. Let $D$ be a digraph $D$ with $\Delta^{+}(D)<n-1$ and $\Delta^{-}(D)<n-1$. What is the minimum size of $D$ when $D$ satisfies WEDCC?

\section{Data Availability}

No data were used to support this study.

\section{Conflicts of Interest}

The author declares no conflicts of interest.

\section{References}

[1] L. Csaba, R. J. Faudree, A. Gyárfás, J. Lehel, and R. H. Schelp, "Networks communicating for each pairing of terminals," Networks, vol. 22, no. 7, pp. 615-626, 1992.
[2] R. J. Faudree, "Properties in path-pairable graphs, New Zealand," Jurnal Matematika, vol. 21, pp. 91-106, 1992.

[3] R. J. Faudree, A. Gyárfás, and J. Lehel, "Minimal path pairable graphs," Congressus Numerantium, vol. 88, pp. 111-128, 1992.

[4] R. J. Faudree, A. Gyárfás, and J. Lehel, "Path-pairable graphs," J. Combin. Math. Comput., pp. 145-157, 1999.

[5] A. S. Jobson, A. E. Kézdy, and J. Lehel, "The minimum size of graphs satisfying cut conditions," Discrete Applied Mathematics, vol. 237, pp. 89-96, 2018.

[6] L. Lovász, Combinatorial Problems and Exercises, AMS Chelsea Publishing, Providence, RI, 2007.

[7] A. Lemple, S. Even, and I. Cederbaum, "An algorithm for planarity testing of graphs," in Proceedings of the Theory of Graphs: International Symposium, P. Rosenstiehl, Ed., Gordon \& Breach, New York, NY, USA, pp. 215-232, July 1966.

[8] G. Fan, B. Xu, X. Yu, and C. Zhou, "Upper bounds on minimum balanced bipartitions," Discrete Mathematics, vol. 312, no. 6, pp. 1077-1083, 2012.

[9] K. Azuma, "Weighted sums of certain dependent random variables," Tokuku Math. J.vol. 19, pp. 357-367, 1967.

[10] W. Hoeffding, "Probability inequalities for sums of bounded random variables," Journal of the American Statistical Association, vol. 58, no. 301, pp. 13-30, 1963.

[11] S. Janson, T. Łuczak, and A. Ruciński, Random Graphs, Wiley, New York, NY, USA, 2000. 